\section{Commentary: New sight, lost sight, insight, and novel sight}

\author{
Alden H. Harken, MD
}

Conceptually, most of us once viewed the mechanism of atrial (and ventricular) fibrillation as a chaotic spectrum of cardiomyocytes depolarizing "automatically" in a nonpredictable (or mappable) fashion. Then Jim Cox proposed a surgical procedure, the success of which depended on isolating segments of the atrium. For the Cox-Maze procedure to be effective, the mechanism of atrial fibrillation must be reentrant. All the data that we have now acquired support the hypothesis that atrial fibrillation is a reentrant rhythm. As we know, reentrant rhythms depend on zones of slow conduction, rapid refractoriness, and unidirectional block. All of this depends on areas of electroanatomic heterogeneity. At one time, it was thought that the reentrant circuits ran around the whole mass of the atria and ventricles; however, it has become apparent that the circuits can be smaller and smaller. As with the programmed stimulation that our colleagues induce in the electrophysiology laboratory, spontaneous atrial and ventricular premature beats can, under proper circumstances of electroanatomic disease, provoke sustained reentrant atrial and ventricular dysrhythmias.

Kharbanda and colleagues ${ }^{1}$ are certainly correct that higher levels of endoepicardial conduction asynchrony are consistent with the electroanatomic heterogeneity

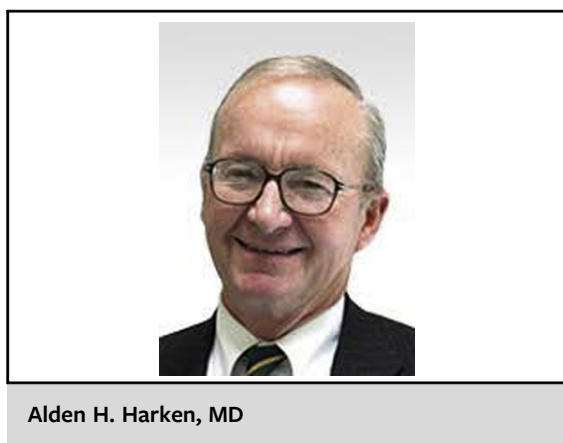

CENTRAL MESSAGE

Our cardiomyocytes and our

national culture share heteroge-

neity as the mechanism foment-

ing our flashpoints.

fundamental to reentrant dysrhythmias. Indeed, cardiomyocyte and cultural disparities are challenging and perhaps inevitable. As surgical electrophysiologists, we must aspire to President Biden's call for the pacification that blossoms with unity. Working together, we can mitigate both dangerous cardiomyocyte and cultural ectopic depolarizations.

\section{Reference}

1. Kharbanda RK, van Schie MS, Taverne YJHJ, de Groot NMS, Bogers AJJC. Novel insights in pathophysiology of postoperative atrial fibrillation. J Thorac Cardiovasc Surg Open. 2021;6:120-9. 